REPRESENTATION THEORY

An Electronic Journal of the American Mathematical Society

Volume 13, Pages 349-370 (August 18, 2009)

S 1088-4165(09)00356-2

\title{
UNITARY REPRESENTATIONS OF RATIONAL CHEREDNIK ALGEBRAS
}

\author{
PAVEL ETINGOF AND EMANUEL STOICA, \\ WITH AN APPENDIX BY STEPHEN GRIFFETH
}

\begin{abstract}
We study unitarity of lowest weight irreducible representations of rational Cherednik algebras. We prove several general results, and use them to determine which lowest weight representations are unitary in a number of cases.

In particular, in type A, we give a full description of the unitarity locus (justified in Subsection 5.1 and the appendix written by S. Griffeth), and resolve a question by Cherednik on the unitarity of the irreducible subrepresentation of the polynomial representation. Also, as a by-product, we establish Kasatani's conjecture in full generality (the previous proof by Enomoto assumes that the parameter $c$ is not a half-integer).
\end{abstract}

\section{INTRODUCTION}

One of the important problems in the theory of group representations is to determine when an irreducible complex representation of a given group is unitary. In the case of noncompact Lie groups, this is a very hard problem, which has not been completely solved. For $p$-adic groups, this problem leads to the difficult and interesting problem of classification of unitary representations of affine Hecke algebras.

In this paper, we begin to study the problem of classification of unitary representations for rational Cherednik algebras. Recall that a rational Cherednik algebra $H_{c}(W, \mathfrak{h})$ is defined by a finite group $W$, a finite dimensional complex representation $\mathfrak{h}$ of $W$, and a function $c$ on conjugacy classes of reflections in $W$. Recall also that for any irreducible representation $\tau$ of $W$, one can define the irreducible lowest weight representation $L_{c}(\tau)$ of $H_{c}(W, \mathfrak{h})$. If $c\left(s^{-1}\right)=\bar{c}(s)$ for all reflections $s$, then the representation $L_{c}(\tau)$ admits a unique, up to scaling, nondegenerate contravariant Hermitian form. We say that $L_{c}(\tau)$ is unitary if this form is positive definite (under an appropriate normalization).

The main problem is then to determine for which $c$ and $\tau$ the representation $L_{c}(\tau)$ is unitary. This problem is motivated by harmonic analysis, and was posed by I. Cherednik. In general, it appears to be quite difficult, like its counterpart in the theory of group representations. The goal of this paper is to begin to attack this problem, by proving a number of partial results about unitary representations.

Received by the editors May 5, 2009 and, in revised form, June 12, 2009.

2000 Mathematics Subject Classification. Primary 16S99.

(C)2009 American Mathematical Society 
More specifically, for every $\tau$ we define the set $U(\tau)$ of values of $c$ for which the representation $L_{c}(\tau)$ is unitary. We prove several general results about $U(\tau)$, and use them to determine the sets $U(\tau)$ in a number of special cases.

In particular, Theorem 5.5 gives a full description of the sets $U(\tau)$ in type A. Namely, it states that unless $\tau$ is the trivial or sign representation (in which case $U(\tau)=(-\infty, 1 / n]$ and $[-1 / n,+\infty)$ respectively), the set $U(\tau)$ consists of the interval $[-1 / \ell, 1 / \ell]$, where $\ell$ is the length of the largest hook of $\tau$ ("the continuous spectrum") and a certain finite set of points of the form $1 / j$, where $j$ are integers ("the discrete spectrum"). We note that the authors of the main body of the paper were unable to prove Theorem 5.5 in its full strength; they were only able to prove that the claimed set contains the unitarity locus, which in turn contains the interval $[-1 / \ell, 1 / \ell]$, and some additional partial results discussed in Subection 5.1. The proof of Theorem 5.5 was completed by an argument due to S. Griffeth, which uses Cherednik's technique of intertwiners and Suzuki's work $[\mathrm{Su}$, and is contained in the appendix.

We also answer, for type $A$, a question by Cherednik, proving that if $c=1 / m$, $2 \leq m \leq n$, then the irreducible submodule $N_{c}$ of the polynomial module $M_{c}(\mathbb{C})$ over the rational Cherednik algebra $H_{c}\left(S_{n}, \mathbb{C}^{n}\right)$ is unitary, and moreover its unitary structure is given by the integration pairing with the Macdonald-Mehta measure.

As a by-product, we determine in full generality the structure of the polynomial representation of the rational Cherednik algebra of type $A$, conjectured by Dunkl $\mathrm{Du}$; this implies a similar description of the structure of the polynomial representation of the double affine Hecke algebra, conjectured by Kasatani [Ka]. These results were established earlier by Enomoto [E] under an additional assumption that $c$ is not a half-integer, which we show to be unnecessary.

The organization of the paper is as follows. Section 2 contains preliminaries. In Section 3, we prove some general properties of unitarity loci, and completely determine them in the rank 1 case. In Section 4 , we focus on the special case of real reflection groups, prove some general properties of the unitarity loci, and compute them in the rank 2 case. In Section 5 we give the results in type A: prove the Dunkl-Kasatani conjecture, answer Cherednik's question, state the theorem on the classification of unitary representations, and begin its proof. The proof is completed in the appendix.

\section{Preliminaries}

2.1. Definition of rational Cherednik algebras. Let $\mathfrak{h}$ be a finite dimensional vector space over $\mathbb{C}$ with a positive definite Hermitian 11 inner product $($,$) . Let T$ : $\mathfrak{h} \rightarrow \mathfrak{h}^{*}$ be the antilinear isomorphism defined by the formula $\left(T y_{1}\right)\left(y_{2}\right)=\left(y_{2}, y_{1}\right)$.

Let $W$ be a finite subgroup of the group of unitary transformations of $\mathfrak{h}$. A reflection in $W$ is an element $s \in W$ such that $\left.\operatorname{rk}(s-1)\right|_{\mathfrak{h}}=1$. Denote by $S$ the set of reflections in $W$. Let $c: S \rightarrow \mathbb{C}$ be a $W$-invariant function. For $s \in S$, let $\alpha_{s} \in \mathfrak{h}^{*}$ be a generator of $\left.\operatorname{Im}(s-1)\right|_{\mathfrak{h}^{*}}$, and $\alpha_{s}^{\vee} \in \mathfrak{h}$ the generator of $\left.\operatorname{Im}(s-1)\right|_{\mathfrak{h}}$, such that $\left(\alpha_{s}, \alpha_{s}^{\vee}\right)=2$. If $W$ is generated by reflections, then we denote by $d_{i}, i=1, \ldots, \operatorname{dim} \mathfrak{h}$, the degrees of the generators of $\mathbb{C}[\mathfrak{h}]^{W}$.

\footnotetext{
${ }^{1}$ We agree that Hermitian forms are antilinear on the second argument.
} 
Definition 2.1 (see e.g. EG, E1]). The rational Cherednik algebra $H_{c}(W, \mathfrak{h})$ is the quotient of the algebra $\mathbb{C} W \ltimes T\left(\mathfrak{h} \oplus \mathfrak{h}^{*}\right)$ by the ideal generated by the relations

$$
\left[x, x^{\prime}\right]=0,\left[y, y^{\prime}\right]=0,[y, x]=(y, x)-\sum_{s \in S} c_{s}\left(y, \alpha_{s}\right)\left(x, \alpha_{s}^{\vee}\right) s,
$$

$x, x^{\prime} \in \mathfrak{h}^{*}, y, y^{\prime} \in \mathfrak{h}$.

An important role in the representation theory of rational Cherednik algebras is played by the element

$$
\mathbf{h}=\sum_{i} x_{i} y_{i}+\frac{\operatorname{dim} \mathfrak{h}}{2}-\sum_{s \in S} \frac{2 c_{s}}{1-\lambda_{s}} s
$$

where $y_{i}$ is a basis of $\mathfrak{h}, x_{i}$ the dual basis of $\mathfrak{h}^{*}$, and $\lambda_{s}$ is the nontrivial eigenvalue of $s$ in $\mathfrak{h}^{*}$. Its usefulness comes from the fact that it satisfies the identities

$$
\left[\mathbf{h}, x_{i}\right]=x_{i},\left[\mathbf{h}, y_{i}\right]=-y_{i} .
$$

2.2. Verma modules, irreducible modules, and the contravariant form. Let $\tau$ be an irreducible representation of $W$. Denote by $M_{c}(\tau)$ the corresponding Verma module, $M_{c}(\tau)=H_{c}(W, \mathfrak{h}) \otimes_{\mathbb{C} W \ltimes S \mathfrak{h}} \tau$, where $\mathfrak{h}$ acts on $\tau$ by zero. Any quotient of $M_{c}(\tau)$ is called a lowest weight module with lowest weight $\tau$. Denote by $L_{c}(\tau)$ the smallest of such modules, i.e., the unique irreducible quotient of the module $M_{c}(\tau)$. If confusion is possible, we will use the long notation $M_{c}(W, \mathfrak{h}, \tau)$, $L_{c}(W, \mathfrak{h}, \tau)$ for $M_{c}(\tau), L_{c}(\tau)$.

Denote by $\mathcal{O}_{c}(W, \mathfrak{h})$ the category of $H_{c}(W, \mathfrak{h})$-modules which are finitely generated under the action of $\mathbb{C}[\mathfrak{h}]$, and locally nilpotent under the action of $\mathfrak{h}$. Examples of objects of this category are $M_{c}(\tau)$ and $L_{c}(\tau)$.

It is easy to see that the element $\mathbf{h}$ acts locally finitely on any object of $\mathcal{O}_{c}(W, \mathfrak{h})$, with finite dimensional generalized eigenspaces. In particular, it acts semisimply on any lowest weight module $M$, with lowest eigenvalue

$$
h_{c}(\tau)=\frac{\operatorname{dim} \mathfrak{h}}{2}-\left.\sum_{s \in S} \frac{2 c_{s}}{1-\lambda_{s}} s\right|_{\tau} .
$$

All other eigenvalues of $\mathbf{h}$ on $M$ are obtained by adding a nonnegative integer to $h_{c}(\tau)$, and this nonnegative integer gives a $\mathbb{Z}_{+}$-grading on $M$.

If $M \in \mathcal{O}_{c}(W, \mathfrak{h})$, then a vector $v \in M$ is called a singular vector if $y v=0$ for any $y \in \mathfrak{h}$. It is clear that a lowest weight module $M$ is irreducible if and only if it has no nonzero singular vectors of positive degree.

2.3. Unitary representations. Let $c^{\dagger}$ be the function on $S$ defined by $c^{\dagger}(s)=$ $\bar{c}\left(s^{-1}\right)$. Fix a $W$-invariant Hermitian form $(,)_{\tau}$ on $\tau$, normalized to be positive definite.

Proposition 2.2. (i) There exists a unique $W$-invariant Hermitian form $\beta_{c, \tau}$ on $M_{c}(\tau)$ which coincides with $(,)_{\tau}$ in degree zero, and satisfies the contravariance condition

$$
\left(y v, v^{\prime}\right)=\left(v, T y \cdot v^{\prime}\right), v, v^{\prime} \in M_{c}(\tau), y \in \mathfrak{h} .
$$

(ii) The kernel of $\beta_{c, \tau}$ coincides with the maximal proper submodule $J_{c}(\tau)$ of $M_{c}(\tau)$, so this form descends to a nondegenerate form on the quotient $M_{c}(\tau) / J_{c}(\tau)$ $=L_{c}(\tau)$.

Proof. Standard. 
We will call $\beta_{c, \tau}$ the contravariant Hermitian form. It is defined uniquely up to a positive scalar, which will not be important.

Let $C$ denote the space of functions $c$ such that $c=c^{\dagger}$.

Definition 2.3. Let $c \in C$. The representation $L_{c}(\tau)$ is said to be unitary if the form $\beta_{c, \tau}$ is positive definite on $L_{c}(\tau)$.

Definition 2.4. $U(\tau)$ is the set of points $c \in C$, such that $L_{c}(\tau)$ is unitary. We call $U(\tau)$ the unitarity locus for $\tau$.

\section{General properties of the sets $U(\tau)$}

\subsection{The general case.}

Proposition 3.1. (i) $U(\tau)$ is a closed set in $C$.

(ii) The point 0 belongs to the interior of $U(\tau)$ for any $\tau$.

(iii) The connected component $Y_{0}(\tau)$ of 0 in the set $Y(\tau)$ of all $c \in C$ for which the form $\beta_{c, \tau}$ is nondegenerate (i.e., $M_{c}(\tau)$ is irreducible) is contained in $U(\tau)$.

(iv) Let $M$ be a lowest weight representation of $H_{c_{0}}(W, \mathfrak{h})$ which is the limit of a 1-parameter family of irreducible unitary representations $L_{c_{0}+t c_{1}}(\tau), t \in(0, \varepsilon)$, as $t$ goes to 0 . Then all composition factors of $M$ are unitary.

Proof. (i) $c \in U(\tau)$ iff the contravariant form is nonnegative definite on $M_{c}(\tau)$, which is a closed condition on $c$.

(ii) We have a natural identification of $M_{c}(\tau)$ with $\tau \otimes \mathbb{C}[\mathfrak{h}]$, and the form $\beta_{0, \tau}$ is the tensor product of the form $(,)_{\tau}$ on $\tau$ and the standard inner product on $\mathbb{C}[\mathfrak{h}]$, given by the formula $(f, g)=\left(D_{g} f\right)(0), D_{g} \in S \mathfrak{h}$ being the differential operator on $\mathfrak{h}$ with constant coefficients corresponding to $g \in S \mathfrak{h}^{*}$ (via the operator $T$ ). Thus $\beta_{0, \tau}>0$, as desired.

(iii) This follows from the standard fact that a continuous family of nondegenerate Hermitian forms is positive definite iff one of them is positive definite.

(iv) This follows from the standard argument with the Jantzen filtration.

It is useful to consider separately the case of constant functions $c \in C$ (in this case $c$ is real). Namely, let $U^{*}(\tau) \subset \mathbb{R}$ be the set of all $c \in \mathbb{R}$ that belong to $U(\tau)$. It is easy to see that analogously to Proposition 3.1, we have:

Corollary 3.2. (i) $U^{*}(\tau)$ is a closed set in $\mathbb{R}$.

(ii) The point 0 belongs to the interior of $U^{*}(\tau)$ for any $\tau$.

(iii) The connected component $Y_{0}^{*}(\tau)$ of 0 in the set $Y^{*}(\tau)$ of all $c \in \mathbb{R}$ for which the form $\beta_{c, \tau}$ is nondegenerate (i.e., $M_{c}(\tau)$ is irreducible) is contained in $U^{*}(\tau)$.

Let $W_{\mathrm{ab}}^{\vee}$ be the group of characters of $W$. It is easy to see that $W_{\mathrm{ab}}^{\vee}$ acts on the space $C$ by multiplication. It also acts on representations of $W$ by tensor multiplication.

Proposition 3.3. For any $\chi \in W_{\mathrm{ab}}^{\vee}$ one has $U(\chi \otimes \tau)=\chi U(\tau)$.

Proof. The statement follows from the fact that we have a natural isomorphism $i_{\chi}: H_{c}(W, \mathfrak{h}) \rightarrow H_{\chi^{-1} c}(W, \mathfrak{h})$ given by the formula $w \rightarrow \chi^{-1}(w) w, w \in W$, and $i_{\chi}(x)=x, i_{\chi}(y)=y, x \in \mathfrak{h}^{*}, y \in \mathfrak{h}$. The pushforward by this isomorphism maps $\tau$ to $\chi \otimes \tau$, which implies the statement.

Proposition 3.4. If $c \in U(\tau)$, then for any irreducible representation $\sigma$ of $W$ that occurs in $\tau \otimes \mathfrak{h}^{*}$, one has $h_{c}(\sigma) \leq h_{c}(\tau)+1$. 
Proof. We will need the following easy lemma (which is probably known, but we give its proof for the reader's convenience).

Lemma 3.5. Let $\sigma \subset \tau \otimes \mathfrak{h}^{*}$ be an irreducible subrepresentation. Let us regard $\sigma$ as sitting in degree 1 of $M_{c}(\tau)$. Then the elements $y_{i}$ act on $\sigma$ by 0 (i.e., $\sigma$ consists of singular vectors) if and only if $h_{c}(\sigma)-h_{c}(\tau)=1$.

Proof. The action of $y_{i}$ on the degree 1 part of $M_{c}(\tau)$ can be viewed as an operator $\tau \otimes \mathfrak{h}^{*} \otimes \mathfrak{h} \rightarrow \tau$, or, equivalently, as an endomorphism $F_{c, \tau, 1}$ of $\tau \otimes \mathfrak{h}^{*}$. This endomorphism is easy to compute, and it is given by the formula

$$
F_{c, \tau, 1}=1-\sum_{s \in S} c_{s} s \otimes\left(\alpha_{s} \otimes \alpha_{s}^{\vee}\right)=1-\sum_{s \in S} \frac{2 c_{s}}{1-\lambda_{s}} s \otimes(1-s) .
$$

Thus $F_{c, \tau, 1}$ acts on $\sigma$ by the scalar

$$
1+h_{c}(\tau)-h_{c}(\sigma) .
$$

The action of $y_{i}$ on $\sigma$ is zero iff this scalar is zero, which implies the lemma.

Now look at the restriction of the form $\beta_{c, \tau}$ to an irreducible $W$-subrepresentation $\sigma$ sitting in the degree 1 part $\tau \otimes \mathfrak{h}^{*}$ of $M_{c}(\tau)$. This restriction is obviously of the form $\ell(c)(,)_{\sigma}$, where $\ell(c)$ is a linear nonhomogeneous function of $c$. Since $\ell(c)$ is positive for $c=0$ (by Proposition 3.1(ii)), we conclude, using Lemma 3.5, that $\ell(c)=K\left(1+h_{c}(\tau)-h_{c}(\sigma)\right)$, where $K>0$. This implies the statement.

Let $D_{\tau}$ be the eigenvalue of $\sum_{s \in S} s$ on $\tau$.

Corollary 3.6. If $c \in U^{*}(\tau)$, then for any $\sigma$ contained in $\tau \otimes \mathfrak{h}^{*}$, one has

$$
c\left(D_{\tau}-D_{\sigma}\right) \leq 1 .
$$

3.2. The operator $F_{c, \tau, m}$. It is useful to generalize the operator $F_{c, \tau, 1}$ acting in degree 1 to higher degrees. Namely, for $c \in C$, we have a unique selfadjoint operator $F_{c, \tau}$ on $M_{c}(\tau)=\tau \otimes S \mathfrak{h}^{*}$, given by the formula $\beta_{c, \tau}\left(v, v^{\prime}\right)=\beta_{0, \tau}\left(F_{c, \tau} v, v^{\prime}\right)$. We have $F_{c, \tau}=\bigoplus_{m>0} F_{c, \tau, m}$, where $F_{c, \tau, m}: \tau \otimes S^{m} \mathfrak{h}^{*} \rightarrow \tau \otimes S^{m} \mathfrak{h}^{*}$ is an operator which is a polynomial in $c$ of degree at most $m$. It is clear that if $F_{c, \tau, m}$ is independent of $c$, then $F_{c, \tau, m}=1$, because $F_{0, \tau, m}=1$. Also, we have the following recursive formula for $F_{c, \tau, m}$ :

Proposition 3.7. Let $a_{1}, \ldots, a_{m} \in \mathfrak{h}^{*}$, and $v \in \tau$. Then

$$
\begin{gathered}
F_{c, \tau, m}\left(a_{1} \ldots a_{m} v\right) \\
=\frac{1}{m} \sum_{j=1}^{m} a_{j} F_{c, \tau, m-1}\left(a_{1} \ldots a_{j-1} a_{j+1} \ldots a_{m} v\right) \\
-\frac{1}{m} \sum_{j=1}^{m} \sum_{s \in S} \frac{2 c_{s}}{1-\lambda_{s}}(1-s)\left(a_{j}\right) F_{c, \tau, m-1}\left(a_{1} \ldots a_{j-1} s\left(a_{j+1} \ldots a_{m} v\right)\right)
\end{gathered}
$$

Remark 3.8. Note that for $m=1$ this formula reduces to formula (2).

Proof. It is easy to see that for any $y \in \mathfrak{h}$, one has

$$
F_{c, \tau, m-1}\left(y a_{1} \ldots a_{m} v\right)=\partial_{y} F_{c, \tau, m}\left(a_{1} \ldots a_{m} v\right) .
$$

Therefore, we find

$$
F_{c, \tau, m}(u)=\frac{1}{m} \sum_{i} x_{i} F_{c, \tau, m-1}\left(y_{i} u\right) .
$$


Taking $u=a_{1} \ldots a_{m} v$ and computing $y_{i} u$ using the commutation relations of the rational Cherednik algebra, we get the result.

Corollary 3.9. Suppose that $F_{c, \tau, i}$ is constant (and hence equals 1 ) for $i=1, \ldots$, $m-1$. Then on every irreducible $W$-subrepresentation $\sigma$ of $\tau \otimes S^{m} \mathfrak{h}^{*}$, the operator $F_{c, \tau, m}$ acts by the scalar $1+\frac{h_{c}(\tau)-h_{c}(\sigma)}{m}$.

3.3. The rank 1 case. Suppose $\mathfrak{h}$ is 1 -dimensional, and $W=\mathbb{Z} / m \mathbb{Z}$, acting by $j \rightarrow \lambda^{-j}$, where $\lambda$ is a primitive $m$-th root of unity. In this case all irreducible representations of $W$ are 1-dimensional, so thanks to Proposition 3.3, to describe the sets $U(\tau)$, it suffices to describe the set $U:=U(\mathbb{C})$ for the trivial representation $\mathbb{C}$. Let us find $U$.

The module $M_{c}(\mathbb{C})$ has basis $x^{n}, n \geq 0$. Let $a_{n}:=\beta_{c, \mathbb{C}}\left(x^{n}, x^{n}\right)$ (we normalize the form so that $\left.a_{0}=1\right)$. It is easy to compute that

$$
a_{n}=a_{n-1}\left(n-2 \sum_{j=1}^{m-1} \frac{1-\lambda^{j n}}{1-\lambda^{j}} c_{j}\right)
$$

where $c_{j}=c(j), j=1, \ldots, m-1$.

Let

$$
b_{n}:=2 \sum_{j=1}^{m-1} \frac{1-\lambda^{j n}}{1-\lambda^{j}} c_{j},
$$

$n \geq 0$ (note that $b_{0}=0$, and $b_{n+m}=b_{n}$ ). If $c \in C$, then $b_{j}$ are real, and it is easy to see that $b_{1}, \ldots, b_{m-1}$ form a linear real coordinate system on $C$ (this follows from the easy fact that the matrix with entries $\frac{1-\lambda^{j n}}{1-\lambda^{j}}, 1 \leq j, n \leq m-1$, is nondegenerate).

This implies the following proposition.

Proposition 3.10. (i) $M_{c}(\mathbb{C})$ is irreducible iff $n-b_{n} \neq 0$ for any $n \geq 1$. It is unitary iff $n-b_{n}>0$ for all $n=1, \ldots, m-1$.

(ii) Assume that for a given c, $r$ is the smallest positive integer such that $r=b_{r}$. Then $L_{c}(\mathbb{C})$ has dimension $r$ (which can be any number not divisible by $m$ ), and basis $1, x, \ldots, x^{r-1}$. This representation can be unitary only if $r<m$, and in this case it is unitary iff $n-b_{n}>0$ for $n<r$.

Corollary 3.11. $U$ is the set of all vectors $\left(b_{1}, \ldots, b_{m-1}\right)$ such that in the vector $\left(1-b_{1}, 2-b_{2}, \ldots, m-1-b_{m-1}\right)$, all the entries preceding the first zero entry are positive (if there is no zero entries, all entries must be positive).

In particular, if $m=2$ and $c_{1}=c$, then $b_{1}=2 c$, and we find that $U=(-\infty, 1 / 2]$ (at the point $c=1 / 2$ the unitary representation is 1 -dimensional).

\section{The Real Reflection CASE}

4.1. The $\mathbf{s l}(2)$ condition. In the rest of the paper, we will assume that $\mathfrak{h}$ is the complexification of a real vector space $\mathfrak{h}_{\mathbb{R}}$ with a positive definite inner product, which is extended to a Hermitian inner product on the complexification, and that $W$ acts by orthogonal transformations on $\mathfrak{h}_{\mathbb{R}}$. Then $s^{2}=1$ for any reflection $s$, and thus $c \in C$ iff $c$ is real valued.

In this case, let us choose $y_{i}$ to be an orthonormal basis of $\mathfrak{h}_{\mathbb{R}}$. Then it is easy to see that

$$
\mathbf{h}=\frac{1}{2} \sum\left(x_{i} y_{i}+y_{i} x_{i}\right),
$$


and that we also have elements

$$
\mathbf{e}=-\frac{1}{2} \sum x_{i}^{2}, \mathbf{f}=\frac{1}{2} \sum y_{i}^{2} .
$$

These elements form an $\mathfrak{s l}_{2}$-triple.

Proposition 4.1. (i) A unitary representation $L_{c}(\tau)$ of $H_{c}(W, \mathfrak{h})$ restricts to a unitary representation of $\mathfrak{s l}_{2}(\mathbb{R})$ from lowest weight category $\mathcal{O}$. In particular, $h_{c}(\tau)=\frac{\operatorname{dim} \mathfrak{h}}{2}-\left.\sum c_{s} s\right|_{\tau} \geq 0$.

(ii) A unitary representation $L_{c}(\tau)$ is finite dimensional iff $L_{c}(\tau)=\tau$.

(iii) An irreducible lowest weight representation $L_{c}(\tau)$ coincides with $\tau$ iff $h_{c}(\sigma)-$ $h_{c}(\tau)=1$ for any irreducible representation $\sigma$ of $W$ contained in $\tau \otimes \mathfrak{h}^{*}$. In this case $h_{c}(\tau)=0$.

Proof. (i) Straightforward.

(ii) If $L_{c}(\tau)$ is finite dimensional, then by (i), it is a trivial representation of $\mathfrak{s l}_{2}(\mathbb{R})$. So $\mathbf{h}=0$, and hence by (1), $x_{i}=0$, which implies the statement.

(iii) The statement $L_{c}(\tau)=\tau$ is equivalent to the statement that $y_{i}$ acts by 0 on any subrepresentation $\sigma$ in $\tau \otimes \mathfrak{h}^{*}$, which by Lemma 3.5] is equivalent to the condition $h_{c}(\sigma)-h_{c}(\tau)=1$.

4.2. Unitarity locus $U^{*}(\tau)$ for exterior powers of the reflection representation. Let $W$ be an irreducible Coxeter group, and $\mathfrak{h}$ its reflection representation. Recall that the representations $\wedge^{i} \mathfrak{h}$ are irreducible. In particular, $\wedge^{\operatorname{dim} \mathfrak{h} h}$ is the sign representation $\mathbb{C}_{-}$of $W$.

Corollary 4.2. (i) For all $\tau$ one has $U^{*}(\tau) \supset[-1 / h, 1 / h]$, where $h$ is the Coxeter number of $W$.

(ii) $U^{*}(\mathbb{C})=(-\infty, 1 / h]$, and $U^{*}\left(\mathbb{C}_{-}\right)=[-1 / h,+\infty)$.

(iii) For $0<i<\operatorname{dim} \mathfrak{h}, U^{*}\left(\wedge^{i} \mathfrak{h}\right)=[-1 / h, 1 / h]$.

Proof. (i) It is known (DJO, GGOR]) that if $c \in(-1 / h, 1 / h)$, then $c$ is a regular value, which means that the category $\mathcal{O}_{c}(W, \mathfrak{h})$ is semisimple. So all $M_{c}(\tau)$ are irreducible, which implies the desired statement by Proposition 3.1(iii).

(ii) Suppose $c \in U^{*}(\mathbb{C})$. We have $h_{c}(\mathbb{C})=\frac{\operatorname{dim} \mathfrak{h}}{2}-c|S|=|S|\left(\frac{1}{h}-c\right)$. Since $h_{c}(\mathbb{C}) \geq 0$, we get $c \leq 1 / h$. On the other hand, for $c<0$ the module $M_{c}(\mathbb{C})$ is irreducible, hence unitary. So the first statement of (ii) follows from (i). The second statement of (ii) follows from the first one by Proposition 3.3 .

(iii) The " $\supset$ " part follows from (i). To prove the " $\subset$ " part, note that the irreducible representation $\wedge^{i+1} \mathfrak{h}$ sits naturally in the degree 1 part of $M_{c}\left(\wedge^{i} \mathfrak{h}\right)$ (as $\mathfrak{h} \otimes \wedge^{i} \mathfrak{h}$ contains a copy of $\left.\wedge^{i+1} \mathfrak{h}\right)$.

Let us compute $D_{\wedge^{i} \mathfrak{h}}$. It is easy to see that the trace of a reflection in $\wedge^{i} \mathfrak{h}$ is

$$
\left(\begin{array}{c}
\operatorname{dim} \mathfrak{h}-1 \\
i
\end{array}\right)-\left(\begin{array}{c}
\operatorname{dim} \mathfrak{h}-1 \\
i-1
\end{array}\right)
$$

Thus, we have

$$
D_{\wedge^{i} \mathfrak{h}}=|S| \frac{\left(\operatorname{dim}_{i}^{\mathfrak{h}-1}\right)-\left(\begin{array}{c}
\operatorname{dim} \mathfrak{h}-1 \\
i-1
\end{array}\right)}{\left(\begin{array}{c}
\operatorname{dim} \mathfrak{h} \\
i
\end{array}\right)}=|S|\left(1-\frac{2 i}{\operatorname{dim} \mathfrak{h}}\right) .
$$

Hence,

$$
h_{c}\left(\wedge^{i+1} \mathfrak{h}\right)-h_{c}\left(\wedge^{i} \mathfrak{h}\right)=2 c|S| / \operatorname{dim} \mathfrak{h}=c h,
$$


So Proposition 3.4 tells us that for any $c \in U^{*}\left(\wedge^{i} \mathfrak{h}\right)$ one has $c h \leq 1$. The rest follows from Proposition 3.3 and part (i).

4.3. The rank 2 case. In this subsection we will calculate the sets $U(\tau)$ in the rank 2 case, i.e., for dihedral groups $W$.

We start with odd dihedral groups. Let $W$ be the dihedral group whose order is $2(2 d+1)$. This group has only one conjugacy class of reflections (so $C=\mathbb{R}$ ), two 1-dimensional representations, $\mathbb{C}$ and $\mathbb{C}_{-}$, and $d$ 2-dimensional representations $\tau_{l}$, $l=1, \ldots, d$, defined by the condition that the counterclockwise rotation by the angle $2 \pi /(2 d+1)$ acts in this representation with eigenvalues $\zeta^{l}$ and $\bar{\zeta}^{l}$, where $\zeta=e^{\frac{2 \pi i}{2 d+1}}$. The reflection representation $\mathfrak{h}$ is thus the representation $\tau_{1}$.

Proposition 4.3. $U(\mathbb{C})=\left(-\infty, \frac{1}{2 d+1}\right], U\left(\mathbb{C}_{-}\right)=\left[-\frac{1}{2 d+1},+\infty\right)$, and $U\left(\tau_{l}\right)=$ $\left[-\frac{l}{2 d+1}, \frac{l}{2 d+1}\right]$ for all $1 \leq l \leq d$.

Proof. The first two statements are special cases of Corollary 4.2(ii), since the Coxeter number $h$ of $W$ is $2 d+1$. To prove the last statement, let us look at the decomposition $S^{k} \tau_{1}=\tau_{k} \oplus \tau_{k-2} \oplus \ldots$ (the last summand is $\mathbb{C}$ if $k$ is even). By tensoring this decomposition with $\tau_{l}$, we notice that we obtain only 2-dimensional summands if $k<l$, while one-dimensional summands make their first appearance only for $k=l$. It follows by induction in $k$, using Corollary 3.9, that the operator $F_{c, \tau_{l}, k}$ is constant in $c$ (and hence equal to 1 ) for $k<l$ (as $h_{c}(\tau)=1$ for any two-dimensional $\tau$ ). Thus, again by Corollary [3.9, $F_{c, \tau_{l}, l}(X)=\left(1 \pm \frac{2 d+1}{l} c\right) X$ if $X$ belongs to the sign, respectively, trivial subrepresentation of $\tau_{l} \otimes S^{l} \tau_{1}$. This implies that if $c \in U\left(\tau_{l}\right)$, then we must have $c \in\left[-\frac{l}{2 d+1}, \frac{l}{2 d+1}\right]$.

It remains to show that $M_{c}\left(\tau_{l}\right)$ is irreducible if $(2 d+1)|c|<l$. This is proved in the paper [Chm, and can also be proved directly, as follows. It follows from the above that $M_{c}\left(\tau_{l}\right)$ contains no singular vectors of degree $<l$. Assume that $c>0$; then any singular vector would be in the sign representation. Let $k \geq l$ be the degree of this vector. Then we get $h_{c}\left(\mathbb{C}_{-}\right)-h_{c}\left(\tau_{l}\right)=k$, which implies that $(2 d+1) c=k \geq l$, as desired. The case of negative $c$ is similar.

Let us now analyse the case of even dihedral group $W$, of order $4 d, d \geq 2$ (the dihedral group of a regular $2 d$-polygon). In this case there are two conjugacy classes of reflections, represented by Coxeter generators $s_{1}, s_{2}$, such that $\left(s_{1} s_{2}\right)^{2 d}=1$. The 1-dimensional representations of $W$ are $\mathbb{C}, \mathbb{C}_{-}$, and also the representations $\varepsilon_{1}$ and $\varepsilon_{2}$, defined by the formulas

$$
\varepsilon_{1}:\left\{\begin{array}{c}
s_{1} \longrightarrow-1, \\
s_{2} \longrightarrow 1,
\end{array} \quad \varepsilon_{2}:\left\{\begin{array}{c}
s_{1} \longrightarrow 1 \\
s_{2} \longrightarrow-1 .
\end{array}\right.\right.
$$

In addition, there are $d-1$ two-dimensional representations $\tau_{l}$, for all $1 \leq l \leq$ $d-1$, given by the same formulas as in the odd case; in particular, as before, $\mathfrak{h}=\tau_{1}$. We will extend the notation $\tau_{l}$ to all integer values of $l$, so that we have $\tau_{l}=\tau_{-l}$ and $\tau_{d-l}=\tau_{d+l}, \tau_{0}=\mathbb{C} \oplus \mathbb{C}_{-}$, and $\tau_{d}=\varepsilon_{1} \oplus \varepsilon_{2}$. Note that $\tau_{l} \otimes \varepsilon_{i}=\tau_{d-l}$ and $\tau_{l} \otimes \mathbb{C}_{-}=\tau_{l}$.

Let $c_{1}$ and $c_{2}$ be the values of the parameter $c$ on the two conjugacy classes of reflections. We will now describe the sets $U(\tau)$ in the plane with coordinates $c_{1}, c_{2}$. By Proposition 3.3. it suffices to find $U(\tau)$ for $\tau=\mathbb{C}$ and $\tau=\tau_{l}, 1 \leq l \leq d-1$.

Proposition 4.4. (i) $U(\mathbb{C})$ is the union of the region defined by the inequalities $c_{1}+c_{2}<\frac{1}{d}, c_{1} \leq \frac{1}{2}$ and $c_{2} \leq \frac{1}{2}$ with the line $c_{1}+c_{2}=\frac{1}{d}$. 
(ii) If $1 \leq l \leq d-1$, then $U\left(\tau_{l}\right)$ is the rectangle defined by the inequalities $\left|c_{1}+c_{2}\right| \leq \frac{l}{d}$ and $\left|c_{1}-c_{2}\right| \leq \frac{d-l}{d}$.

Proof. (i) The operator $F_{c, \mathbb{C}, 1}$ is the scalar $1-\left(c_{1}+c_{2}\right) d$. This implies the condition $c_{1}+c_{2} \leq \frac{1}{d}$ for $c \in U(\mathbb{C})$.

Now recall that $S^{k} \tau_{1}=\tau_{k} \oplus \tau_{k-2} \oplus \cdots$ (the last summand is $\mathbb{C}$ for even $k$ ). In particular, $S^{d} \tau_{1}$ contains $\varepsilon_{1}$ and $\varepsilon_{2}$, one copy of each. Consider the operator $F_{c, \mathbb{C}}$ restricted to the subrepresentation $\varepsilon_{i}$. We claim that this operator (which is a scalar, since it is defined on a one-dimensional space) equals

$$
Q(c)=\left(1-2 c_{i}\right) \prod_{j=1}^{d-1}\left(1-\frac{d}{j}\left(c_{1}+c_{2}\right)\right) .
$$

Indeed, it follows from the paper Chm that $\varepsilon_{i}$ consists of singular vectors if $c_{i}=$ $1 / 2$, and that at the line $c_{1}+c_{2}=\frac{l}{d}, 1 \leq l \leq d-1$, there is a singular vector of degree $l$ in the representation $\tau_{l}$, such that the subrepresentation generated by this vector contains $\varepsilon_{i}$ in degree $d$. This implies that $Q(c)$ is divisible by the right-hand side of (3). On the other hand, the degree of $Q(c)$ is $d$, and $Q(0)=1$, which implies (3).

Formula (3) and the inequality $c_{1}+c_{2} \leq 1 / d$ implies that if a unitary representation $L_{c}(\mathbb{C})$ contains $\varepsilon_{i}$ in degree $d$, then we must have $c_{i}<1 / 2$. It remains to consider unitary representations $L_{c}(\mathbb{C})$ that do not contain $\varepsilon_{i}$ for some $i$. This means that either this $\varepsilon_{i}$ is singular in $M_{c}(\mathbb{C})$ (which means $c_{i}=1 / 2$ ) or the copy of $\tau_{1}$ in degree 1 is singular, i.e., $c_{1}+c_{2}=1 / d$. This proves part (i).

(ii) Assume that $l<d / 2$. Similarly to the case of odd dihedral group, there is no 1-dimensional representations in $M_{c}\left(\tau_{l}\right)$ in degrees $k<l$, while the trivial and sign representations sit in degree $l$. As in the odd case, this implies, by using induction in $k$ and Corollary 3.9 that $F_{c, \tau_{l}, i}=1$ for $i<l$, and $F_{c, \tau_{l}, l}(X)=\left(1 \pm \frac{d}{l}\left(c_{1}+c_{2}\right)\right) X$ if $X$ belongs to the sign, respectively, trivial subrepresentation of $\tau_{l} \otimes S^{l} \tau_{1}$. This implies that if $c \in U(\tau)$, then $\left|c_{1}+c_{2}\right| \leq \frac{l}{d}$.

Let us now prove the second inequality $\left|c_{1}-c_{2}\right| \leq \frac{d-l}{d}$. By Chm, we have singular vectors living in $\varepsilon_{1}$ and $\varepsilon_{2}$ in degree $d-l$. Since $S^{d-2 l} \tau_{1}$ does not contain 1-dimensional representations, by using the same argument as in the proof of Proposition 3.9. we conclude that $F_{c, \tau_{l}, d-l}$ acts on $\varepsilon_{i}$ by the scalars $1 \pm \frac{d}{d-l}\left(c_{1}-c_{2}\right)$, which proves the desired inequality for unitary representations.

Finally, if both inequalities are satisfied strictly, then it follows from $\mathrm{Chm}$ that $M_{c}\left(\tau_{l}\right)$ is irreducible, and thus the rectangle defined by these inequalities is contained in $U\left(\tau_{l}\right)$, as desired.

If $l \geq d / 2$, the result is obtained by applying Proposition 3.3 to $\chi=\varepsilon_{1}$. Part (ii) is proved.

4.4. The Gaussian inner product. Part (ii) of Proposition 4.1 can be generalized. For this purpose we want to introduce the Gaussian inner product on any lowest weight representation $M$ of $H_{c}(W, \mathfrak{h})$, which was defined by Cherednik ([Ch1]).

Definition 4.5. The Gaussian inner product $\gamma_{c, \tau}$ on $M_{c}(\tau)$ is given by the formula

$$
\gamma_{c, \tau}\left(v, v^{\prime}\right)=\beta_{c, \tau}\left(\exp (\mathbf{f}) v, \exp (\mathbf{f}) v^{\prime}\right) .
$$

This makes sense because the operator $\mathbf{f}$ is locally nilpotent on $M_{c}(\tau)$. Thus we see that $\gamma_{c, \tau}$ has kernel $J_{c}(\tau)$, so it descends to an inner product on any lowest 
weight module with lowest weight $\tau$, in particular, to a nondegenerate inner product on $L_{c}(\tau)$, and it is positive definite on $L_{c}(\tau)$ if and only if $\beta_{c, \tau}$ is also. The difference between $\beta$ and $\gamma$ is that vectors of different degrees are orthogonal under $\beta$, but not necessarily under $\gamma$.

Proposition 4.6. (i) The form $\gamma_{c, \tau}$ on a lowest weight module $M$ satisfies the condition

$$
\gamma_{c, \tau}\left(x v, v^{\prime}\right)=\gamma_{c, \tau}\left(v, x v^{\prime}\right), x \in \mathfrak{h}_{\mathbb{R}}^{*} .
$$

(ii) Up to scaling, $\gamma_{c, \tau}$ is the unique $W$-invariant form satisfying the condition

$$
\gamma_{c, \tau}\left((-y+T y) v, v^{\prime}\right)=\gamma_{c, \tau}\left(v, y v^{\prime}\right), y \in \mathfrak{h}_{\mathbb{R}} .
$$

Proof. (i) We have

$$
\begin{gathered}
\gamma_{c, \tau}\left(x v, v^{\prime}\right)=\beta_{c, \tau}\left(\exp (\mathbf{f}) x v, \exp (\mathbf{f}) v^{\prime}\right) \\
=\beta_{c, \tau}\left(\left(x+T^{-1} x\right) \exp (\mathbf{f}) v, \exp (\mathbf{f}) v^{\prime}\right)=\beta_{c, \tau}\left(\exp (\mathbf{f}) v,\left(T^{-1} x+x\right) \exp (\mathbf{f}) v^{\prime}\right) \\
=\beta_{c, \tau}\left(\exp (\mathbf{f}) v, \exp (\mathbf{f}) x v^{\prime}\right)=\gamma_{c, \tau}\left(v, x v^{\prime}\right)
\end{gathered}
$$

(ii) A similar computation to (i) yields that the required property holds. Let us now show uniqueness. If $\gamma$ is any $W$-invariant Hermitian form satisfying the condition of (ii), then let $\beta\left(v, v^{\prime}\right)=\gamma\left(\exp (-\mathbf{f}) v, \exp (-\mathbf{f}) v^{\prime}\right)$. Then $\beta$ is contravariant, so by Proposition 2.2 , it is a multiple of $\beta_{c, \tau}$, hence $\gamma$ is a multiple of $\gamma_{c, \tau}$.

Corollary 4.7. Let $c \in U(\tau)$, and let $I_{c}(\tau) \subset \mathbb{C}[\mathfrak{h}]$ be the annihilator of $L_{c}(\tau)$ in $\mathbb{C}[\mathfrak{h}]$. Then $I_{c}(\tau)$ is a radical ideal.

Proof. Assume that $g^{2} \in I_{c}(\tau)$. Then $g^{2} \bar{g}^{2} \in I_{c}(\tau)$, so for any $v \in L_{c}(\tau), \gamma_{c, \tau}\left(g^{2} \bar{g}^{2} v, v\right)$ $=0$. So by Proposition 4.6, $\gamma_{c, \tau}(g \bar{g} v, g \bar{g} v)=0$. Hence $g \bar{g} v=0$, so $\gamma_{c, \tau}(g \bar{g} v, v)=0$, i.e., $\gamma_{c, \tau}(g v, g v)=0$, which implies that $g v=0$, hence $g \in I_{c}(\tau)$.

This corollary is clearly a generalization of Proposition 4.1(ii).

Corollary 4.8. Let $c$ be a constant function, and $c \in U(\tau)$. If $I_{c}(\tau) \neq 0$ (i.e. the support of $L_{c}(\tau)$ is not equal $\mathfrak{h}$, and has smaller dimension), then $c=1 / m$, where $m$ is an integer.

Proof. We will use the results of [BE]. Consider the support $X \subset \mathfrak{h}$ of $L_{c}(\tau)$. By our assumption, $X \neq \mathfrak{h}$. Let $b \in X$ be a generic point. Consider the restriction $N=\operatorname{Res}_{b}\left(L_{c}(\tau)\right)$ defined in $[\mathrm{BE}$. Then $N$ is a finite dimensional irreducible module over $H_{c}\left(W_{b}, \mathfrak{h} / \mathfrak{h}^{W_{b}}\right)$. Moreover, by Corollary 4.7. $I_{c}(\tau)$ is a radical ideal, which implies that $N=N_{c}(\xi)=\xi$ for some irreducible module $\xi$ of $W_{b}$. Therefore, we have $c\left(D_{\sigma}-D_{\xi}\right)=1$, where $D_{\psi}$ is the eigenvalue of $\sum_{s \in S \cap W_{b}} s$ on an irreducible representation $\psi$ of $W_{b}$, and $\sigma$ is an irreducible subrepresentation of $\xi \otimes\left(\mathfrak{h} / \mathfrak{h}^{W_{b}}\right)$. This implies that the numerator of $c$ is 1 , as desired.

4.5. Integral representation of the Gaussian inner product on $M_{c}(\mathbb{C})$. We will need the following known result (see Du2], Theorem 3.10).

Proposition 4.9. We have

$$
\gamma_{c, \mathbb{C}}(f, g)=K(c)^{-1} \int_{\mathfrak{h}_{\mathbb{R}}} f(z) \overline{g(z)} d \mu_{c}(z)
$$

where

$$
d \mu_{c}(z):=e^{-|z|^{2} / 2} \prod_{s \in S}\left|\alpha_{s}(z)\right|^{-2 c_{s}} d z
$$


and

$$
K(c)=\int_{\mathfrak{h}_{\mathbb{R}}} d \mu_{c}(z),
$$

provided that the integral (5) is absolutely convergent.

Proof. It follows from Proposition 4.6 that $\gamma_{c, \tau}$ is uniquely, up to scaling, determined by the condition that it is $W$-invariant, and $y_{i}^{\dagger}=x_{i}-y_{i}$. These properties are easy to check for the right-hand side of (4), using the fact that the action of $y_{i}$ is given by Dunkl operators.

Remark 4.10. As usual, the integral formula extends analytically to arbitrary complex $c$.

Remark 4.11. The constant $K(c)$ is given by the Macdonald-Mehta product formula, proved by E. Opdam Op for Weyl groups and by F. Garvan for $H_{3}$ and $H_{4}$ (for the dihedral groups the formula follows from the beta integral). For an irreducible reflection group $W$ and a constant $c$, this formula has the form

$$
K(c)=K_{0} \prod_{j=1}^{\operatorname{dim} \mathfrak{h}} \frac{\Gamma\left(1-d_{j} c\right)}{\Gamma(1-c)},
$$

where $d_{j}$ are the degrees of $W$. It follows from this formula that for constant $c$ the first pole of $K(c)$ occurs at $c=1 / h$, which gives another proof of Corollary 4.2(ii).

4.6. The simple submodule of the polynomial representation. Let $c$ be a constant positive function, which is a singular value for $W$ (i.e., it is rational and has denominator dividing one of the degrees $d_{i}$ of $W$ ). Let $N_{c}$ be the minimal nonzero submodule of the polynomial representation $M_{c}(\mathbb{C})$. This is an irreducible module of the form $L_{c}\left(\tau_{c}\right)$, where $\tau_{c}$ is a certain irreducible representation of $W$ depending on $c$. It is easy to see that $N_{c}$ is the unique simple submodule of the polynomial representation.

The following observation was made by I. Cherednik.

Proposition 4.12. Suppose that $N_{c}$ is contained in $L^{2}\left(\mathfrak{h}_{\mathbb{R}}, d \mu_{c}\right)$. Then $N_{c}$ is a unitary representation.

Proof. As in the proof of Proposition 4.9, we see that the integral gives a $W$ invariant form $\gamma$ on $N_{c}$ which satisfies the condition $y_{i}^{\dagger}=x_{i}-y_{i}$. By Proposition 4.6. this implies that $\gamma$ is a multiple of $\gamma_{c, \tau_{c}}$. Also, it is manifestly positive definite, as desired.

Motivated by this observation and a number of examples, Cherednik asked the following question.

Question 4.13 ([Ch1]). Let $W$ be an irreducible Coxeter group, $\mathfrak{h}$ its reflection representation, and $c=1 / d_{i}$. Is it true that $N_{c}$ is contained in $L^{2}\left(\mathfrak{h}_{\mathbb{R}}, d \mu_{c}\right)$ ? In particular, is $N_{c}$ unitary?

In the next section we will show that the answer to both questions is "yes" in type A. 


\section{TYPe A}

5.1. The main theorem. In this section we restrict ourselves to the case $W=S_{n}$, $n \geq 2$, and $\mathfrak{h}=\mathbb{C}^{n}$. In this case we have only one conjugacy class of reflections, so $C=\mathbb{R}$. Irreducible representations $\tau$ of $S_{n}$ are labeled by Young diagrams (=partitions); for instance, the trivial representation is $(n)$ (the 1-row diagram) and the sign representation is $\left(1^{n}\right)$ (the 1 -column diagram). We will denote the conjugate partition to a partition $\tau$ by $\tau^{*}$; the corresponding operation on representations is tensoring with the sign representation. Abusing notation, we will denote partitions, Young diagrams, and representations of $S_{n}$ by the same letter (say, $\tau$ ).

We let $\ell(\tau)$ be the length of the largest hook of the Young diagram $\tau, m_{*}(\tau)$ denote the multiplicity of the largest part of $\tau$, and set

$$
N(\tau)=\ell(\tau)-m_{*}(\tau)+1
$$

The eigenvalue $D_{\tau}$ of $\sum_{s \in S} s$ on $\tau$ equals the content $\operatorname{ct}(\tau)$ of the Young diagram $\tau$, i.e., the sum of the numbers $i-j$ over the cells $(i, j)$ of the diagram. Therefore,

$$
h_{c}(\tau)=\frac{n}{2}-c \cdot \operatorname{ct}(\tau)
$$

Proposition 5.1. For a partition $\tau \neq\left(1^{n}\right)$ and each $c \in U(\tau), c \leq \frac{1}{N(\tau)}$.

Proof. Recall that $\tau \otimes \mathfrak{h}^{*}$ is the sum of representations corresponding to Young diagrams $\lambda$ obtained from $\tau$ by removing and adding a corner cell. Also, let $\nu(\tau)$ be the number of parts of $\tau$. Then it is easy to see that $N(\tau)$ is the largest value of $\nu(\tau)+i-j$ over all corner cells $(i, j)$ of the Young diagram $\tau$ (i.e., cells for which neither $(i, j+1)$ nor $(i+1, j)$ belong to $\tau)$. Therefore, the proposition follows from Corollary 3.6

Proposition 5.2. The interval $\left[-\frac{1}{\ell(\tau)}, \frac{1}{\ell(\tau)}\right]$ is contained in $U(\tau)$.

Proof. Let $q=e^{2 \pi i c}, \mathcal{H}_{n}(q)$ be the Hecke algebra of $S_{n}$ with parameter $q$, and let $S^{\tau}$ be the Specht module over $\mathcal{H}_{n}(q)$ corresponding to $\tau$, defined in [DJ1. Then it follows from [DJ2, Theorem 4.11, that $S^{\tau}$ is irreducible if $c \in\left(-\frac{1}{\ell(\tau)}, \frac{1}{\ell(\tau)}\right)$. By the theory of the KZ functor, GGOR, this implies that $M_{c}(\tau)$ is irreducible in this range. This implies the required statement.

Corollary 5.3. If $\tau$ and $\tau^{*}$ contain a part equal to 1 , then $U(\tau)=\left[-\frac{1}{\ell(\tau)}, \frac{1}{\ell(\tau)}\right]$.

Proof. This follows from Propositions 5.1 and 5.2, since if $\tau^{*}$ contains a part equal 1 , then $N(\tau)=\ell(\tau)$.

Proposition 5.4. Let $\tau=(p, p, \ldots, p)$, where $p$ is a divisor of $n$. Then $L_{1 / p}(\tau)$ is unitary.

Proof. This is shown in the proof of Theorem 8.8 in CEE.

The main result of this subsection is the following theorem.

Theorem 5.5. For any $\tau \neq(n),\left(1^{n}\right), U(\tau)$ is the union of the interval $\left[-\frac{1}{\ell(\tau)}, \frac{1}{\ell(\tau)}\right]$ with the finite set of isolated points $\frac{1}{k}$, for $N(\tau) \leq k<l(\tau)$ and $-\ell\left(\tau^{*}\right)<k \leq$ $-N\left(\tau^{*}\right)$ (so there are $m_{*}(\tau)-1$ positive points, and $m_{*}\left(\tau^{*}\right)-1$ negative points).

The proof of Theorem 5.5 starts in this subsection, and is completed in the appendix. 
Theorem 5.6. For any $\tau \neq(n),\left(1^{n}\right), U(\tau)$ is contained in the set defined in Theorem 5.5 .

Proof. Let $N=N(\tau), \ell=\ell(\tau), m_{*}=m_{*}(\tau)$ (so $\ell=N+m_{*}-1$ ). By Propositions 5.1] and 5.2. our job is to show that the intervals $I_{k}=\left(\frac{1}{N+k}, \frac{1}{N+k-1}\right), k=1, \ldots, m_{*}-$ 1 do not intersect with $U(\tau)$.

Denote by $\tau_{i}, i=1, \ldots, m_{*}$ the partition of $n$ obtained by reducing $i$ copies of the largest part of $\tau$ by 1 , and adding $i$ copies of the part 1 . Then it follows from the rule of tensoring by $\mathfrak{h}^{*}$ that $\tau \otimes S^{i} \mathfrak{h}^{*}$ contains a unique copy of $\tau_{i}$. This implies that for any $c, M_{c}(\tau)$ contains a unique copy of $\tau_{i}$ in degree $i$. We have $\left.\beta_{c, \tau}\right|_{\tau_{i}}=f_{i, \tau}(c)(,)_{\tau_{i}}$, where $f$ is a scalar polynomial.

Lemma 5.7. One has, up to scaling:

$$
f_{\tau, i}(c)=(1-(N+i-1) c) \ldots(1-N c) .
$$

Proof. The proof is by induction in $i$. For the base we can take the case $i=0$, which is trivial. To make the inductive step, assume that the statement is proved for $i=m-1$ and let us prove it for $i=m$. By the induction assumption, at $c=\frac{1}{N+j-1}, j=1, \ldots, m-1$, the module $M_{c}(\tau)$ has a singular vector $u$ sitting in $\tau_{j}$ in degree $j$. Indeed, the contravariant form on $\tau_{j}$ is zero at such $c$, and there can be no singular vectors of lower degree, because if one moves $i<j$ corner cells of $\tau$ to get a partition $\sigma$, then $D_{\tau}-D_{\sigma} \leq i(N+i-1)<i(N+j-1)$, so $c\left(D_{\tau}-D_{\sigma}\right)<i$.

Since $\tau \otimes S^{m} \mathfrak{h}^{*}$ contains $\tau_{j} \otimes S^{m-j} \mathfrak{h}^{*}$, which in turn contains $\tau_{m}$, we see that the submodule generated by the singular vector $u$ contains the copy of $\tau_{m}$ in degree $m$, which implies that $f_{\tau, m}$ is divisible by $f_{\tau, m-1}$.

Thus, to complete the induction step, it suffices to show that

$$
f_{\tau, m}^{\prime}(0)=f_{\tau, m-1}^{\prime}(0)-N-m+1 .
$$

To prove this formula, let us differentiate the equation of Proposition 3.7 with respect to $c$ at $c=0$. We get

$$
\begin{gathered}
F_{0, \tau, m}^{\prime}\left(a_{1} \ldots a_{m} v\right) \\
=\frac{1}{m} \sum_{j=1}^{m}\left(a_{j} F_{0, \tau, m-1}^{\prime}\left(a_{1} \ldots a_{j-1} a_{j+1} \ldots a_{m} v\right)-\sum_{s \in S}\left[a_{1} \ldots a_{m}, s\right] v\right) .
\end{gathered}
$$

This can be rewritten, using tensor notation, as follows:

$$
F_{0, \tau, m}^{\prime}=\frac{1}{m} \sum_{j=1}^{m}\left(F_{0, \tau, m-1}^{\prime}\right)_{\hat{j}}-\frac{1}{m}\left(D_{\tau}-D_{\tau_{m}}\right),
$$

where the subscript $\hat{j}$ means that the operator acts in all components of the tensor product but the $j$-th. Since $\tau_{m} \subset \tau_{m-1} \otimes \mathfrak{h}^{*} \subset \tau \otimes S^{m} \mathfrak{h}^{*}$, this equation implies

$$
f_{\tau, m}^{\prime}(0)=f_{\tau, m-1}^{\prime}(0)-\frac{1}{m}\left(D_{\tau}-D_{\tau_{m}}\right)=f_{\tau, m-1}^{\prime}(0)-N-m+1,
$$

as desired.

Now the theorem follows easily from Lemma 5.7. Namely, we see that $L_{c}(\tau)$ is not unitary on the interval $I_{k}$ because the polynomial $f_{\tau, k+1}(c)$ is negative on this interval, and hence the form $\beta_{c, \tau}$ is negative definite on $\tau_{k+1}$. 
Remark 5.8. It follows from GGOR that a module $L_{c}(\tau)$ is thin (i.e., is killed by the $\mathrm{KZ}$ functor or, equivalently, has support strictly smaller than $\mathfrak{h}$ ) if and only if $\tau$ is not $m$-regular, where $m$ is the denominator of $c$ (i.e., it contains some part at least $m$ times). On the other hand, it is easy to show directly by looking at Young diagrams that if $\tau$ is not $m$-regular, and $m \geq N(\tau)$, then $\tau$ is a rectangular diagram, $\tau=(p, \ldots, p)$, and $m=N(\tau)=p$. Thus Theorem [5.6 implies that the representations of Proposition 5.4 are the only thin unitary representations for $c>0$ (and a similar statement is valid for $c<0$ ).

The following result is a special case of Theorem [5.5 but was known before Theorem [5.5] was proved; here we give its original proof.

Theorem 5.9. Let $m=m_{*}(\tau)$, and $\tau_{m}$ be the diagram obtained from $\tau$ by removing the last column and concatenating it with the first one (as in the proof of Theorem 5.6). Then $\frac{1}{\ell(\tau)} \in U\left(\tau_{m}\right)$. In particular, Theorem 5.5 holds if the multiplicity $p=p_{*}(\tau)$ of the part 1 in $\tau$ satisfies the inequality $p \geq m$.

Proof. Since $\left[-\frac{1}{\ell(\tau)}, \frac{1}{\ell(\tau)}\right] \subset U(\tau)$, and (as was shown in the proof of Theorem [5.6) $M_{c}(\tau)$ contains a singular vector in $\tau_{m}$ at $c=1 / \ell(\tau)$, the theorem follows from Proposition 3.1(iv).

5.2. The Dunkl-Kasatani conjecture. The following theorem was conjectured (and partially proved) by Dunkl (see the end of [Du] ) in 2005. It is also the rational version of Kasatani's conjecture for double affine Hecke algebras ( $\mathrm{Ka}$, Conjecture 6.4 ), which was proposed at approximately the same time. In the case when $c \notin \frac{1}{2}+$ $\mathbb{Z}$, this theorem was proved by Enomoto [En] in 2006, using the results of Rouquier on the connection between rational Cherednik algebras and $q$-Schur algebras, and the theory of crystal bases for quantum affine algebras. Enomoto also explained that this theorem implies Kasatani's conjecture. We give a different proof of this theorem, based on the work $[\mathrm{BE}]$, which does not need the condition $c \notin \frac{1}{2}+\mathbb{Z}$.

Theorem 5.10. (i) Assume that $c=r / m$, where $r \geq 1, m \geq 2$ are integers with $(r, m)=1$. Then the module $M_{c}(\mathbb{C})$ has length $l+1$, where $l=[n / m]$. Namely, it has a strictly increasing filtration by submodules,

$$
0=I_{c}^{0} \subset I_{c}^{1} \subset \cdots \subset I_{c}^{l+1}=M_{c}(\mathbb{C}),
$$

such that the successive quotients are irreducible. In particular, $I_{c}^{1}=N_{c}$.

(ii) For $1 \leq j \leq l+1, I_{c}^{j}$ is a lowest weight representation, and its lowest weight is the representation of the symmetric group corresponding to the partition $\tau_{c}^{j}=\left(j m-1, m-1, \ldots, m-1, s_{j}\right)$, where $n-(j-1) m=q_{j}(m-1)+s_{j}, 0 \leq s_{j}<m-1$ if $j \leq l$, and $\tau_{c}^{l+1}=(n)$.

(iii) The variety $V\left(I_{c}^{j}\right) \subset \mathbb{C}^{n}$ defined by the ideal $I_{c}^{j}, j=0, \ldots, l+1$ is the variety $X_{m}^{j}$ of all vectors $\left(x_{1}, \ldots, x_{n}\right)$ which, up to a permutation, have the form

$$
\left(x_{1}, \ldots, x_{n-j m}, a_{1}, \ldots, a_{1}, a_{2}, \ldots, a_{2}, \ldots, a_{j}, \ldots, a_{j}\right),
$$

where each $a_{i}$ is repeated $m$ times (here we agree that $X_{m}^{l+1}=\emptyset$ ).

(iv) $I_{c}^{j}$ are radical ideals if and only if $r=1$.

(v) At the point $c=r / m$, the forms $\beta$ and $\gamma$ have a zero of order exactly $l-j+1$ on the ideal $I_{c}^{j}$ for $j=1, \ldots, l+1$.

Remark 5.11. The ideals of Theorem 5.14 are rational limits of the ideals defined in [FJMM]; see also Ka. 
Proof. Let us first construct the ideals $I_{c}^{j}$. Assume first that $c=1 / m$ (i.e., $r=1$ ). In this case, define $I_{c}^{j}$ to be the defining ideals of the varieties $X_{m}^{j}$. We claim that these ideals are invariant under the Dunkl operators $D_{i}$, i.e., are submodules under the rational Cherednik algebra. To check this, let $f \in I_{c}^{j}$, and $U$ be the formal neighborhood in $\mathbb{C}^{n}$ of the $S_{n}$-orbit of a generic point $u \in X_{m}^{j}$. It is sufficient to show that $D_{i} f=0$ on the intersection $X_{m}^{j} \cap U$. But this follows easily (using the ideology of $[\mathrm{BE}]$ ) from the fact that the irreducible representation $L_{c}\left(S_{m}, \mathbb{C}^{m-1}, \mathbb{C}\right)$ is 1-dimensional (so that the ideal of zero is a subrepresentation of the polynomial representation $M_{c}\left(S_{m}, \mathbb{C}^{m-1}, \mathbb{C}\right)$ ).

The case of $c=r / m$ for a general $r$ such that $(r, m)=1$ is slightly more complicated but similar. Namely, let $I_{r, m}$ be the maximal proper subrepresentation in the polynomial representation $M_{r / m}\left(S_{m}, \mathbb{C}^{m}, \mathbb{C}\right)$. We define $I_{c}^{j}$ to be the intersection of the $S_{n}$-images of the ideal $\mathbb{C}\left[x_{1}, \ldots, x_{n-j m}\right] \otimes I_{r, m}^{\otimes j}$. Then the same argument as above shows that $I_{c}^{j}$ are a nested sequence of subrepresentations of the polynomial representation $M_{c}(\mathbb{C})$. Moreover, since the representation $L_{c}\left(S_{m}, \mathbb{C}^{m-1}, \mathbb{C}\right)$ is finite dimensional, the variety defined by the ideal $I_{c}^{j}$ is $X_{m}^{j}$. Also, it is easy to see from the definition that $I_{c}^{j}$ is a radical ideal if and only if $r=1$. Thus, we have proved (iii) and (iv).

To prove the rest of the theorem, we need the following lemma.

Lemma 5.12. The length of the polynomial representation $M_{c}(\mathbb{C})$ is $l+1$, and its composition factors are $L_{c}\left(\tau_{c}^{j}\right), j=1, \ldots, l+1$.

Proof. It is shown in $[\mathrm{Du}]$ that $M_{c}(\mathbb{C})$ has singular vectors living in $\tau_{c}^{j}$, so these irreducible representations do occur in the composition series, so that the length of $M_{c}(\mathbb{C})$ is at least $l+1$.

We prove that the length is in fact exactly $l+1$ (i.e., no other composition factors occur) by induction in $n$. The base of induction is trivial, so we only need to justify the inductive step. For this purpose, let $b \in \mathbb{C}^{n}$ be a point with stabilizer $S_{n-1}$, and consider the restriction functor $\operatorname{Res}_{b}: \mathcal{O}_{c}\left(S_{n}, \mathbb{C}^{n}\right) \rightarrow \mathcal{O}_{c}\left(S_{n-1}, \mathbb{C}^{n}\right)$ defined in [BE]. This functor is exact. Moreover, the support of any simple object in $\mathcal{O}_{c}\left(S_{n}, \mathbb{C}^{n}\right)$ is $X_{m}^{j}$ for some $j, 0 \leq j \leq l$, so if $n$ is not divisible by $m$, the functor $\operatorname{Res}_{b}$ does not kill any simple objects (as $b \in X_{m}^{j}$ for all $j$ in this case). This implies that

length $\left(M_{c}\left(S_{n}, \mathbb{C}^{n}, \mathbb{C}\right)\right) \leq$ length $\left(\operatorname{Res}_{b}\left(M_{c}\left(S_{n}, \mathbb{C}^{n}, \mathbb{C}\right)\right)\right)=\operatorname{length}\left(M_{c}\left(S_{n-1}, \mathbb{C}^{n}, \mathbb{C}\right)\right)$.

But length $\left(M_{c}\left(S_{n-1}, \mathbb{C}^{n}, \mathbb{C}\right)\right)=l+1$ by the induction assumption, so we are done.

It remains to consider the case when $n=m l$. In this case, we have

$$
\operatorname{length}\left(M_{c}\left(S_{n-1}, \mathbb{C}^{n}, \mathbb{C}\right)\right)=l,
$$

which is even better for us, but the problem is that now the functor $\operatorname{Res}_{b}$ may (and actually does) kill simple objects, as $b \notin X_{m}^{l}$. However, we still have $b \in X_{m}^{j}$, $j<l$, so the above argument shows that the composition series of $M_{c}\left(S_{n}, \mathbb{C}^{n}, \mathbb{C}\right)$ is as desired, plus possibly some additional simple modules supported on the variety $X_{m}^{l}$ (the smallest of all $X_{m}^{j}$ ). So to prove the induction step (i.e., show that in fact there is no additional modules), it suffices to show that the composition series of $M_{c}\left(S_{n}, \mathbb{C}^{n}, \mathbb{C}\right)$ contains at most one simple module supported on $X_{m}^{l}$.

To do so, consider a point $b \in \mathbb{C}^{n}$ with stabilizer $\left(S_{m}\right)^{l}$, and the corresponding functor $\operatorname{Res}_{b}: \mathcal{O}_{c}\left(S_{n}, \mathbb{C}^{n}\right) \rightarrow \mathcal{O}_{c}\left(\left(S_{m}\right)^{l}, \mathbb{C}^{n}\right)$. This functor is exact and does not kill any simple objects, as $b \in X_{m}^{j}$ for all $j, 0 \leq j \leq l$. Thus, it suffices to show that in the composition series of $\operatorname{Res}_{b}\left(M_{c}\left(S_{n}, \mathbb{C} n, \mathbb{C}\right)\right.$ ) (or, equivalently, of 
$\left.M_{c}\left(\left(S_{m}\right)^{l}, \mathbb{C}^{n}, \mathbb{C}\right)=M_{c}\left(S_{m}, \mathbb{C}^{m}, \mathbb{C}\right)^{\otimes l}\right)$ there is at most one simple object with support of dimension $l$. So it is enough to check that in the composition series of $M_{c}\left(S_{m}, \mathbb{C}^{m}, \mathbb{C}\right)$ there is at most one simple object with support of dimension 1 (and all other terms have supports of larger dimension), i.e., that in the composition series of $M_{c}\left(S_{m}, \mathbb{C}^{m-1}, \mathbb{C}\right)$ there is at most one finite dimensional simple object. But it is well known (see [BEG]) that this composition series involves only two simple objects, only one of which is finite dimenional.

Now we finish the proof of Theorem 5.10. Lemma 5.12 implies that the quotient $I_{c}^{j+1} / I_{c}^{j}$ is irreducible for each $j$. Also, the support of this representation is $X_{m}^{j}$. So since by $\left[\mathrm{BE}\right.$, the support of $L_{c}\left(\tau_{c}^{j}\right)$ is also $X_{m}^{j}$, we find that $I_{c}^{j+1} / I_{c}^{j}=L_{c}\left(\tau_{c}^{j}\right)$.

Lemma 5.13. Any submodule $E$ of $M_{c}(\mathbb{C})$ coincides with $I_{c}^{j}$ for some $j$.

Proof. Let $X$ be the variety defined by $E$. Then $X=X_{m}^{j}$ for some $j$ (by $\mathrm{BE}$, Section 3.8). So $M_{c}(\mathbb{C}) / E$ may involve in its Jordan-Holder series only $L_{c}\left(\tau_{c}^{i}\right)$ with $i>j$. This means that $E \supset I_{c}^{j}$. On the other hand, restricting to a generic point of $X_{m}^{j}$ (as in $\left[\mathrm{BE}\right.$ ), we see that we must have $E \subset I_{c}^{j}$. This implies $E=I_{c}^{j}$, as desired.

Finally, recall again from $\left[\mathrm{Du}\right.$ that $M_{c}(\mathbb{C})$ contains singular vectors in representations $\tau_{c}^{j}, j=1, \ldots, l+1$. Let $W_{j}$ be the highest weight submodules of $M_{c}(\mathbb{C})$ generated by these singular vectors. The unique irreducible quotient of $W_{j}$ is $L_{c}\left(\tau_{c}^{j}\right)$, so by the above we must have $W_{j}=I_{c}^{j}$. In particular, $I_{c}^{j}=W_{j}$ are a nested sequence of lowest weight modules, as anticipated in $\mathrm{Du}$, Section 6 .

We have now established (i) and (ii). To establish the remaining statement (v), it suffices to observe that it follows from (i)-(iv) that the Jantzen filtration on $M_{c}(\mathbb{C})$ coincides with the filtration by the ideals $I_{c}^{j} 2$

\subsection{Unitarity of the irreducible subrepresentation of the polynomial rep- resentation.}

Theorem 5.14. Let $W=S_{n}, \mathfrak{h}=\mathbb{C}^{n}$, and $2 \leq m \leq n$. Then $N_{1 / m} \subset \mathbb{C}\left[x_{1}, \ldots, x_{n}\right]$ is contained in $L^{2}\left(\mathfrak{h}_{\mathbb{R}}, d \mu_{c}\right)$. In particular, $N_{1 / m}$ is unitary. Thus, the answer to Cherednik's Question 4.13 for type $A$ is affirmative.

Remark 5.15. Note that the statement that $N_{1 / m}$ is unitary in Theorem 5.14 is a special case of Theorem 5.5 (taking into account Theorem [5.10(ii)).

Proof. Let $P \in \mathbb{C}\left[x_{1}, \ldots, x_{n}\right]$, and set

$$
\xi_{P}(c)=\int_{\mathbb{R}^{n}}|P(z)|^{2} d \mu_{c}(z) .
$$

It is a standard fact that $\xi_{P}$ is a holomorphic function of $c$ for $\operatorname{Re} c \leq 0$ which extends meromorphically to the whole complex plane. By Proposition 4.9, $\xi_{P}(c)=$ $K(c) \gamma_{c, \mathbb{C}}(P, P)$, which implies that the poles of $\xi_{P}(c)$ may occur only for $c=r / m>$ 0 , where $2 \leq m \leq n$ and $(r, m)=1$, and the order of such a pole is at most $[n / m]+1$ (which is the order of the pole of $K(c)$ at $c=r / m$ ). In fact, it is clear from Theorem 5.10(v) that the order of the pole of $\xi_{P}(c)$ at $c=r / m$ is at most $j-1$ if $P \in I_{c}^{j}$, $j>0$. In particular, there is no pole of $\xi_{c}(P)$ for $c=r / m$ if $P \in N_{r / m}$.

The proof is based on the following proposition.

\footnotetext{
${ }^{2}$ This fact is discussed in Ch2], p. 15, and also in [Ch1].
} 
Proposition 5.16. If $P \in N_{1 / m}, 2 \leq m \leq n$, then $\xi_{P}(c)$ has no poles for $c<\frac{1}{m-1}$.

This proposition implies Theorem 5.14 Indeed, it implies that for $c<\frac{1}{m-1}$, $P \in L^{2}\left(\mathbb{R}^{n}, d \mu_{c}\right)$.

Proof of Proposition 5.16. Let $\frac{r}{k}<\frac{1}{m-1},(r, k)=1$, so $r(m-1)<k \leq n$. Our job is to show that $N_{1 / m} \subset N_{r / k}$, so that $\xi_{P}(c)$ has no pole at $c=r / k$.

Let $S_{c}$ be the scheme defined by the ideal $N_{c}$. We have to show that $S_{r / k} \subset S_{1 / m}$.

By Theorem 5.10(iii,iv), $S_{1 / m}$ is the reduced scheme (variety) $X_{m}^{1}$, which is the set of all points $\left(x_{1}, \ldots, x_{n}\right)$ such that some $m$ coordinates coincide with each other. The scheme $S_{r / k}$ is not necessarily reduced, but by Theorem 5.10(iii), the underlying variety $\bar{S}_{r / k}$ is $X_{k}^{1}$.

By Theorem 5.10 (i), $N_{r / k}$ is the set of all $f \in \mathbb{C}\left[x_{1}, \ldots, x_{n}\right]$ such that $f$ vanishes in the formal neighborhood in $S_{r / k}$ of a generic point of $X_{k}^{1}$, i.e., a point $u=$ $\left(x_{1}, \ldots, x_{n}\right)$ where some $k$ coordinates coincide with each other, and there is no other coincidences. Therefore, it suffices to check that for any $f \in N_{1 / m}, f$ vanishes on the formal neighborhood in $S_{r / k}$ of $u$. For this, it suffices to check that this holds if $f$ belongs to the lowest weight subspace $Q$ of $N_{1 / m}$. By using [BE] and restricting to the formal neighborhood of $u$, we see that it is sufficient to show that the representation $Q$, regarded as a representation of $S_{k}$, is disjoint from the representation $L_{r / k}\left(S_{k}, \mathbb{C}^{k-1}, \mathbb{C}\right)$ regarded as an $S_{k}$-module (i.e., there is no nontrivial homomorphisms between these two representations).

Now, we know from Theorem 5.10(ii) that the lowest weight subspace $Q$ is the representation of $S_{n}$ corresponding to the Young diagram $\tau_{m}^{1}=(m-1, \ldots, m-1, s)$, where $n=q(m-1)+s$. Also, recall from [BEG that $L_{r / k}\left(S_{k}, \mathbb{C}^{k-1}, \mathbb{C}\right)$, as a representation of $S_{k}$, is the space of complex functions on the group $A \subset(\mathbb{Z} / r \mathbb{Z})^{k}$ of vectors with zero sum of coordinates. Such a vector has a coordinate $i \in \mathbb{Z} / r \mathbb{Z}$ with multiplicity $n_{i}$, and $\sum n_{i}=k$. So irreducible representations of $S_{n}$ that occur in $L_{r / k}\left(S_{k}, \mathbb{C}^{k-1}, \mathbb{C}\right)$ are those representations $Y$ for which $Y^{S_{n_{1}} \times \cdots \times S_{n_{r}}} \neq 0$ for some $n_{1}, \ldots, n_{r}$ such that $n_{1}+\cdots+n_{r}=k$.

However, we claim that $Q^{S_{n_{1}} \times \cdots \times S_{n_{r}}}=0$ for any $n_{1}, \ldots, n_{r}$ with $n_{1}+\cdots+n_{r}=k$. Indeed, it is standard that $Q$ is generated by the polynomial $P:=\Delta_{q+1}^{\otimes s} \otimes \Delta_{q}^{\otimes m-1-s}$, where $\Delta_{p}$ is the Vandermonde determinant in $p$ variables. On the other hand, since $r(m-1)<k$, we have $m-1<n_{i}$ for some $i$. This means that if we symmetrize $P$ with respect to any conjugate of the subgroup $S_{n_{i}}$, we get zero, as desired.

Theorem 5.14 is proved.

\section{Appendix: Proof of Theorem 5.5}

\section{by Stephen Griffeth}

The purpose of this appendix is to apply the results in Suzuki's paper $[\mathrm{Su}$, which classifies and describes those irreducible modules in category $\mathcal{O}$ on which the Cherednik-Dunkl subalgebra acts diagonalizably to the problem of unitarity of $L_{c}(\tau)$. In Theorem 3.7.2 of his book [Ch3], I. Cherednik proved results analogous to Suzuki's for the double affine Hecke algebra of type $A$ and it would be interesting to apply them to classify the unitary modules for the double affine Hecke algebra.

We will use the definitions and notation of Sections 1-5 of the present paper, except as noted in this paragraph. In order to conform with Suzuki's notation, we set $\kappa=-1 / c$, and write $\mathbb{H}_{\kappa}$ for the rational Cherednik algebra attached to $S_{n}$ 
acting on its permutation representation. Let $y_{1}, \ldots, y_{n}$ be the standard basis of the permutation representation $\mathfrak{h}=\mathbb{C}^{n}$ of $S_{n}$ and let $x_{1}, \ldots, x_{n}$ be the dual basis of $\mathfrak{h}^{*}$. As in $\mathrm{Su}$ the commutation relation for $y_{i}$ and $x_{j}$ is

$$
y_{i} x_{j}= \begin{cases}x_{j} y_{i}-s_{i j} & \text { if } i \neq j, \text { and } \\ x_{i} y_{i}+\kappa+\sum_{k \neq i} s_{i k} & \text { if } i=j .\end{cases}
$$

This differs from the relations used in Section 5 of this paper only by a scaling that does not affect the question of unitarity. We write $L_{\kappa}(\tau)$ for the irreducible representation corresponding to a partition $\tau$. For the remainder of the paper we assume that $\kappa \in \mathbb{Q}$.

The Cherednik-Dunkl subalgebra of $\mathbb{H}_{\kappa}$ is generated by the elements

$$
z_{i}=y_{i} x_{i}-\phi_{i} \quad \text { where } \quad \phi_{i}=\sum_{1 \leq j<i} s_{i j} .
$$

The elements $z_{1}, \ldots, z_{n}$ are pairwise commutative. Let $w_{0} \in S_{n}$ be the longest element, with $w_{0}(i)=n-i+1$ for $1 \leq i \leq n$. By the defining relations for $\mathbb{H}_{\kappa}$,

$$
\begin{aligned}
w_{0} z_{i} w_{0}^{-1} & =y_{n-i+1} x_{n-i+1}-\sum_{1 \leq j<i} s_{n-i+1, n-j+1} \\
& =x_{n-i+1} y_{n-i+1}+\kappa+\sum_{j \neq n-i+1} s_{n-i+1, j}-\sum_{1 \leq j<i} s_{n-i+1, n-j+1} \\
& =\epsilon_{n-i+1}^{\vee}+\kappa
\end{aligned}
$$

where as in Proposition 4.2 of Suzuki's paper [Su],

$$
\epsilon_{i}^{\vee}=x_{i} y_{i}+\sum_{1 \leq j<i} s_{i j}
$$

Note that $z_{i}$ and $\epsilon_{i}^{\vee}$ are invariant with respect to the anti-automorphism $*$, and hence act diagonalizably on the unitary modules $L_{\kappa}(\tau)$. Suzuki's paper describes the irreducible modules in category $\mathcal{O}$ for $\mathbb{H}_{\kappa}$ on which $\epsilon_{1}^{\vee}, \ldots, \epsilon_{n}^{\vee}$ (or equivalently $\left.z_{1}, \ldots, z_{n}\right)$ act diagonalizably and gives an explicit combinatorial description of their weight space decompositions.

We will need the intertwining operators

$$
\sigma_{i}=s_{i}-\frac{1}{z_{i}-z_{i+1}}, \Phi=x_{n} s_{n-1} \cdots s_{1} \text {, and } \Psi=y_{1} s_{1} \cdots s_{n-1},
$$

the fact that they map eigenvectors for $z_{1}, \ldots, z_{n}$ to eigenvectors, and the formulas

$$
\begin{gathered}
\sigma_{i}^{2}=\frac{\left(z_{i}-z_{i+1}\right)^{2}-1}{\left(z_{i}-z_{i+1}\right)^{2}}, \quad \Psi \Phi=z_{1}, \\
\sigma_{i}^{*}=\sigma_{i} \text { and } \Phi^{*}=\Psi
\end{gathered}
$$

where $*$ is the conjugate linear anti-automorphism of $\mathbb{H}_{\kappa}$ with $x^{*}=T x, y^{*}=T y$, and $w^{*}=w^{-1}$ for $x \in \mathfrak{h}, y \in \mathfrak{h}$, and $w \in S_{n}$. For any $f \in \mathbb{H}_{\kappa}, f^{*}$ is the adjoint of $f$ with respect to the contravariant form on $L_{\kappa}(\tau)$. All of this is by now standard; proofs of the more general facts for the groups $G(r, 1, n)$ may be found in Gri1.

Let $\tau$ be a partition of $n$ of length $m$ and let $\kappa \in \mathbb{Q}>0$. We regard $\tau$ as the subset of $\mathbb{Z} \times \mathbb{Q}$ containing the points $(i, j)$ for $i, j \in \mathbb{Z}$ with $1 \leq i \leq m$ and $1 \leq j \leq \tau_{i}$. Put $p=(-m, \kappa-m)$ and let $\widehat{\tau}$ be the subset of $\mathbb{Z} \times \mathbb{Q}$ given by

$$
\widehat{\tau}=\tau+\mathbb{Z} p \text {. }
$$


It contains $\tau$ as a subset. A periodic tableau on $\widehat{\tau}$ is a bijection $T: \widehat{\tau} \rightarrow \mathbb{Z}$ such that

$$
\text { for all } b \in \widehat{\tau} \text {, we have } T(b+p)=T(b)-n
$$

and a periodic tableau $T$ is standard if for $(a, b) \in \widehat{\tau}$ such that $(a, b+1) \in \widehat{\tau}$, we have

$$
T(a, b)<T(a, b+1)
$$

and for $(a, b) \in \widehat{\tau}$ and $k \in \mathbb{Z}_{\geq 0}$ with $(a+k+1, b+k) \in \widehat{\tau}$ we have

$$
T(a, b)<T(a+k+1, b+k) .
$$

The content vector of the tableau $T$ is

$$
\operatorname{ct}(T)=\left(\operatorname{ct}\left(T^{-1}(1)\right), \ldots, \operatorname{ct}\left(T^{-1}(n)\right)\right), \quad \text { where } \operatorname{ct}(a, b)=b-a .
$$

When $\kappa \in \mathbb{Z}$ we will think of $\tau$ and $\widehat{\tau}$ as consisting of collections of boxes, and a tableau as a filling of those boxes with integers.

Having fixed $n \in \mathbb{Z}_{>0}$, write $P$ for the set of integer partitions of $n$. Let $\kappa \in \mathbb{Q}_{>0}$, and write $\kappa=s / r$ with $r, s \in \mathbb{Z}_{>0}$ and $(r, s)=1$. Recall the definition of $N(\tau)$ from the second paragraph of Section 5.1 and define

$$
P_{\kappa}=\left\{\tau \in P \mid s \geq N\left(\tau^{*}\right)\right\} \quad \text { where } \tau^{*} \text { is the transpose of } \tau \text {. }
$$

Write

$$
S(\widehat{\tau})=\{\text { standard tableaux } T \text { on } \widehat{\tau} \text { with } T(b)>0 \text { for } b \in \tau\} .
$$

Now we combine Theorems 4.8 and 4.12 of Suzuki's paper $\mathrm{Su}$ into the following theorem:

Theorem 6.1 (Suzuki). The set of diagonalizable irreducible $\mathbb{H}_{\kappa}$-modules in $\mathcal{O}$ is $\left\{L_{\kappa}(\tau) \mid \tau \in P_{\kappa}\right\}$, and for $\tau \in P_{\kappa}$ we have

$$
L_{\kappa}(\tau)=\bigoplus_{T \in S(\widehat{\tau})} L_{\kappa}(\tau)_{c t(T)}, \quad \text { with } \quad \operatorname{dim}_{\mathbb{C}}\left(L_{\kappa}(\tau)_{c t(T)}\right)=1 \text { for all } T \in S(\widehat{\tau}),
$$

where for a sequence $a_{1}, \ldots, a_{n}$ of numbers

$$
L_{\kappa}(\tau)_{\left(a_{1}, \ldots, a_{n}\right)}=\left\{m \in L(\tau) \mid \epsilon_{i}^{\vee} \cdot m=a_{i} m \text { for } 1 \leq i \leq n\right\} .
$$

We now finish the proof of Theorem 5.5 started in Section 5. We rewrite the statement of Theorem 5.5 in terms of $\kappa=-1 / c$ so that it becomes

$$
\begin{aligned}
\left\{\kappa \mid L_{\kappa}(\tau) \text { is unitary }\right\} & =\left\{\kappa \in \mathbb{Z}_{>0} \mid \kappa \geq N\left(\tau^{*}\right)\right\} \\
& \cup\left\{\kappa \in \mathbb{Z}_{<0} \mid \kappa \leq-N(\tau)\right\} \\
& \cup\{\kappa \in \mathbb{Q}|| \kappa \mid \geq \ell(\tau)\} .
\end{aligned}
$$

This will follow from the results of Section 5 and Theorem 6.3 below. First we prove a preparatory lemma.

Recall that $\mathfrak{t}$ is the Cherednik-Dunkl subalgebra generated by $z_{1}, \ldots, z_{n}$. A weight of $\mathfrak{t}$ on a module $M$ is thus described by the sequence $\alpha=\left(\alpha_{1}, \ldots, \alpha_{n}\right)$ of complex numbers giving the action of $z_{1}, \ldots, z_{n}$.

Lemma 6.2. The module $L_{\kappa}(\tau)$ is unitary exactly if it is $\mathbf{t}$-diagonalizable and for all weights $\alpha=\left(\alpha_{1}, \ldots, \alpha_{n}\right)$ of $\mathfrak{t}$ on $L_{\kappa}(\tau)$,

$$
\alpha_{1} \geq 0 \quad \text { and } \quad\left(\alpha_{i}-\alpha_{i+1}\right)^{2} \geq 1
$$


Proof. The module $L_{\kappa}(\tau)$ is graded by finite dimensional subspaces, and $\mathfrak{t}$ preserves these subspaces. The contravariant form is unitary on $L_{\kappa}(\tau)$ exactly if its restriction to each graded piece is also.

Suppose first that $L_{\kappa}(\tau)$ is unitary. Then $z_{1}, \ldots, z_{n}$ act on each graded piece as commuting self-adjoint operators, and it follows that $L_{\kappa}(\tau)$ is $\mathfrak{t}$-diagonalizable. For a t-eigenvector $f \in L_{\kappa}(\tau)$ of weight $\alpha$, one has

$$
\langle\Phi . f, \Phi . f\rangle=\langle f, \Psi \Phi . f\rangle=\left\langle f, z_{1} . f\right\rangle=\alpha_{1}\langle f, f\rangle
$$

and it follows that $\alpha_{1} \geq 0$. Similarly, for $1 \leq i \leq n-1$,

$$
\left\langle\sigma_{i} . f, \sigma_{i} . f\right\rangle=\left\langle f, \sigma_{i}^{2} . f\right\rangle=\frac{\left(\alpha_{i}-\alpha_{i+1}\right)^{2}-1}{\left(\alpha_{i}-\alpha_{i+1}\right)^{2}}\langle f, f\rangle
$$

and it follows that $\left(\alpha_{i}-\alpha_{i+1}\right)^{2} \geq 1$.

Conversely, the irreducibility of $L_{\kappa}(\tau)$ implies that each weight vector of $\mathfrak{t}$ can be obtained from a weight vector in $S^{\tau} \subseteq L_{\kappa}(\tau)$ by applying a sequence of operators from the set $\left\{\Phi, \sigma_{1}, \ldots, \sigma_{n-1}\right\}$. Assuming that $L_{\kappa}(\tau)$ is diagonalizable and that (19) holds, the equalities (20) and (21) imply that the t-eigenvectors in $L(\tau)$ have nonnegative norms. Therefore Suzuki's theorem and the orthogonality of distinct weight spaces implies that $L_{\kappa}(\tau)$ is unitary.

The lemma reduces the question of unitarity of irreducible lowest weight modules for the rational Cherednik algebra of type $S_{n}$ to an examination of their $\mathfrak{t}$-spectra. One could prove Theorem 5.6 this way. We will use it to finish the proof of the converse:

Theorem 6.3. If $\kappa \in \mathbb{Z}$ and $\kappa \geq N\left(\tau^{*}\right)$ or $\kappa \leq-N(\tau)$, then $L_{\kappa}(\tau)$ is unitary.

Proof. We will show using Lemma 6.2 that if $\kappa \in \mathbb{Z}$ and $\kappa \geq N\left(\tau^{*}\right)$, then $L_{\kappa}(\tau)$ is unitary. Proposition 3.3 then completes the proof. Since $\kappa \geq N\left(\tau^{*}\right)$, Suzuki's Theorem 6.1 implies that the module $L_{\kappa}(\tau)$ is diagonalizable with weight basis $f_{T}$ (for $\mathfrak{t}$ ) in bijection with the set $S(\widehat{\tau})$ of standard tableaux $T$ on $\hat{\tau}=\tau+\mathbb{Z}(-m, \kappa-m)$ such that $T(b)>0$ for $b \in \tau$, where $m$ is the length of $\tau$. The weight of $f_{T}$ is given by

$$
z_{i} \cdot f_{T}=\left(\operatorname{ct}\left(T^{-1}(n-i+1)\right)+\kappa\right) f_{T} \quad \text { for } 1 \leq i \leq n .
$$

The contents of the boxes of $\widehat{\tau}$ are all integers since $\kappa$ is an integer. This implies the second inequality in (19) of Lemma 6.2. Furthermore, adding $\kappa$ to the content of the box containing $n$ gives a nonnegative integer by the definitions of $N\left(\tau^{*}\right)$ and the set $S(\widehat{\tau})$. This implies the first inequality of (19) of Lemma 6.2. The theorem is proved.

By using the same techniques and the results of Gri2, one should be able to determine the set of unitary lowest weight irreducibles for rational Cherednik algebras attached to the groups $G(r, p, n)$. The missing ingredient is the analog of Theorem 6.1 for the groups $G(r, 1, n)$. We are currently working on this problem, using the results of [Gri2] as a starting point. 


\section{ACKNOWLEDGMENTS}

We are very grateful to I. Cherednik, who posed the main problem and suggested a number of important techniques. This paper would not have appeared without his influence. We also thank D. Vogan for many useful discussions about unitary representations of Lie groups, and Charles Dunkl for comments on a preliminary version of the paper. The work of P.E. was partially supported by the NSF grant DMS-0504847. The work of S.G. was partially supported by NSF Career Grant DMS-0449102.

\section{REFERENCES}

[BE] Roman Bezrukavnikov, Pavel Etingof, Parabolic induction and restriction functors for rational Cherednik algebras, arXiv:0803.3639.

[BEG] Yu. Berest, P. Etingof, V. Ginzburg, Finite dimensional representations of rational Cherednik algebras, Int. Math. Res. Not. 19 (2003), 1053-1088. MR.1961261 (2004h:16027)

[CEE] D. Calaque, B. Enriquez, P. Etingof, Universal KZB equations I: the elliptic case, arXiv:math/0702670.

[Ch1] I. Cherednik, Towards harmonic analysis for DAHA: integral formulas for canonical traces, talk, www-math.mit.edu/ etingof/hadaha.pdf.

[Ch2] I. Cherednik, Non-semisimple Macdonald polynomials, arXiv:0709.1742.

[Ch3] I. Cherednik, Double affine Hecke algebras. London Mathematical Society Lecture Note Series, 319. Cambridge University Press, Cambridge, 2005. MR.2133033 (2007e:32012)

[Chm] Chmutova, Tatyana, Representations of the rational Cherednik algebras of dihedral type. J. Algebra 297 (2006), no. 2, 542-565. MR2209274 (2006m:16038)

[DJ1] R. Dipper and G. D. James, Representations of Hecke algebras of the general linear groups, Proc. London Math. Soc. 52 (1986), 20-52. MR812444 (88b:20065)

[DJ2] Richard Dipper, Gordon James, Blocks and idempotents of Hecke algebras of general linear groups, Proc. London Math. Soc. (3) 54 (1987), no. 1, 57-82. MR872250|(88m:20084)

[DJO] C.F. Dunkl, M.F.E. de Jeu and E.M. Opdam, Singular polynomials for finite reflection groups, Trans. Amer. Math. Soc. 346 (1994), 237-256. MR.1273532 (96b:33012)

[Du] C. Dunkl, Singular polynomials and modules for the symmetric groups, math/0501494, Int. Math. Res. Not. 2005, v. 39, 2409-2436. MR2181357 (2006j:33012)

[Du2] C. Dunkl, Integral kernels with reflection group invariance, Can. J. Math. 43 (1991), 1213-1227. MR1145585 (93g:33012)

[EG] P. Etingof, V. Ginzburg, Symplectic reflection algebras, Calogero-Moser space, and deformed Harish-Chandra homomorphism, Invent. Math., vol. 147, (2002), no. 2, 243-348. MR.1881922 (2003b:16021)

[E1] P. Etingof, Calogero-Moser Systems and Representation Theory, Zurich lectures in advanced mathematics European Mathematical Society, 2007. MR.2296754

[En] N. Enomoto, Composition factors of polynomial representation of DAHA and crystallized decomposition numbers, math.RT/0604369.

[FJMM] B. Feigin, M. Jimbo, T. Miwa, E. Mukhin, Symmetric polynomials vanishing on the shifted diagonals and Macdonald polynomials. MR.1962014(2004g:05149)

[Gri1] S. Griffeth, Towards a combinatorial representation theory for the rational Cherednik algebra of type $G(r, p, n)$., to appear in Proceedings of the Edinburgh Mathematical Society. arXiv:math/0612733

[Gri2] S. Griffeth, Orthogonal functions generalizing Jack polynomials, arXiv:0707.0251

[GGOR] V. Ginzburg, N. Guay, E. Opdam, R. Rouquier, On the category $\mathcal{O}$ for rational Cherednik algebras. Invent. Math. 154 (2003), no. 3, 617-651. MR2018786 (2005f:20010)

[Ka] M. Kasatani, Subrepresentations in the polynomial representation of the double affine Hecke algebra of type $G L_{n}$ at $t^{k+1} q^{r-1}=1$, Int. Math. Res. Notices, 2005, no. 28, 1717-1742. MR2172339 (2007c:20012) 
[Op] E. M. Opdam, Some applications of hypergeometric shift operators. Invent. Math. 98 (1989), no. 1, 1-18. MR1010152 (91h:33024)

[Su] T. Suzuki, Cylindrical Combinatorics and Representations of Cherednik Algebras of type A, arXiv:math/0610029.

Department of Mathematics, Room 2-176, Massachusetts Institute of Technology, 77 Massachusetts Avenue, Cambridge, Massachusetts 02139

E-mail address: etingof@math.mit.edu

Department of Mathematics, Room 2-089, Massachusetts Institute of Technology, 77 Massachusetts Avenue, Cambridge, Massachusetts 02139

E-mail address: immanuel@math.mit.edu

School of Mathematics, University of Minnesota, 127 Vincent Hall, 206 Church St. S.E., Minneapolis, Minnesota 55455

E-mail address: griffeth@math.umn.edu 